\title{
Regulation of corrosion damage of magnesium alloys through the use of vacuum zirconium coatings
}

\author{
M. L. Linderov ${ }^{\dagger, 1}$, M. A. Afanasyev ${ }^{1}$, A. N. Asmolov², V. A. Danilov ${ }^{1}$, D. L. Merson ${ }^{1}$ \\ †dartvi@gmail.com \\ ${ }^{1}$ Institute of Advanced Technologies, Togliatti State University, Togliatti, 445020, Russia \\ ${ }^{2}$ Department of Aircraft Production and Quality Control in Mechanical Engineering, Samara National Research University, \\ Samara, 443086, Russia
}

\begin{abstract}
Magnesium alloys have a unique combination of properties in terms of specific strength, modulus of elasticity and biocompatibility, which allows them to be considered as one of the most promising materials for use as temporary bioresorbable implants capable of being replaced by bone tissue in the process of gradual dissolution in the human body, thereby eliminating the need for repeated surgical intervention to remove the implant after healing. In this regard, the world scientific community pays much attention to works aimed at developing methods for controlling the corrosion damage of magnesium alloys. The present study demonstrates that such a precision control can be realized using vacuum zirconium coatings, while $\mathrm{Zr}$ itself is already actively used in medicine. It has been shown that the application of a zirconium coating on the MA14 (ZK60) alloy, which is currently considered as one of the possible candidates for use in medical practice, with thicknesses of 0.4 and $0.8 \mu \mathrm{m}$, can reduce the corrosion damage estimated by the hydrogen yield, based on 110 hours per 1.3 and 1.6 times, respectively. The favorable effect of the zirconium coating on the resistance to corrosion processes is confirmed by the results obtained using a laser confocal microscope: a decrease in both the total corroded area and the depth of corrosion damage was recorded in three-dimensional images of the sample surface. In addition, in the work using scanning electron microscopy, a comprehensive study of the obtained coatings is presented, which made it possible to characterize its structure and chemical composition. The adhesion characteristics of the coating were evaluated by scratching on a tester and showed that for a coating with a thickness of $0.8 \mu \mathrm{m}$, fracture is observed at an indentation load of the conical indenter of the order of $0.5 \mathrm{~N}$.
\end{abstract}

Keywords: magnesium alloys, corrosion, coating, biodegradable material.

УДК: 538.951

\section{Регулирование коррозионной повреждаемости магниевых сплавов за счет использования вакуумных циркониевых покрытий}

\author{
Линдеров М. Л., ${ }^{\dagger}$, Афанасьев М. А. ${ }^{1}$, Асмолов А. Н. ${ }^{2}$, Данилов В. А. ${ }^{1}$, Мерсон Д. Л. ${ }^{1}$ \\ ${ }^{1}$ Лаборатория физики прочности и интеллектуальные диагностические системы, \\ Тольяттинский Государственный Университет, Тольятти, 445020, Россия \\ ${ }^{2}$ Кафедра производства летательных аппаратов и управления качеством в машиностроении, \\ Самарский национальный исследовательский университет им. акад. С. П. Королева, Самара, 443086, Россия
}

\begin{abstract}
Магниевые сплавы обладают уникальным сочетанием свойств по удельной прочности, модулю упругости и биосовместимости, что позволяет их рассматривать как одни из наиболее перспективных материалов для использования в качестве временных биорезорбируемых имплантатов, способных в процессе постепенного растворения в организме человека замещаться костной тканью, тем самым исключая необходимость повторного операционное вмешательства для извлечения имплантата после залечивания. В связи с этим в мировом научном сообществе уделяется много внимания работам, направленным на разработку способов регулирования коррозионной повреждаемости магниевых сплавов. В представленном исследовании продемонстрировано, что такое прецизионное регулирование может быть реализовано за счет использования вакуумных циркониевых
\end{abstract}


покрытий, в то время как сам Zr уже активно используется в медицине. Показано, что нанесение циркониевого покрытия на сплав MA14 (ZK60), который в настоящее время рассматривается как один из возможных кандидатов на использование в медицинской практике, толщинами 0.4 и 0.8 мкм способно уменьшить коррозионную повреждаемость, оцененную по выходу водорода, на базе 110 часов в 1.3 и 1.6 раза, соответственно. Благоприятное влияние циркониевого покрытия на сопротивление коррозионным процессам подтверждается результатами, полученными с использованием лазерного конфокального микроскопа: на трехмерных изображениях поверхности образцов зафиксировано уменьшение как общей прокорродировавшей площади, так и глубины коррозионных повреждений. Кроме того, в работе с использованием сканирующей электронной микроскопии приведено комплексное исследование полученных покрытий, позволившее охарактеризовать его структуру и химический состав. Адгезионные характеристики покрытия оценивались путем царапания на скретч-тестере и показали, что для покрытия толщиной 0.8 мкм разрушение наблюдается при нагрузке вдавливания конического индентора порядка $0.5 \mathrm{H}$.

Ключевые слова: магниевые сплавы, коррозия, покрытие, биорезорбируемый материал.

\section{1. Введение}

В настоящее время магниевые сплавы рассматриваются, как одни из наиболее перспективных материалов для применения в качестве биорезорбируемых имплантатов в медицинской промышленности $[1,2]$. Это связано с их уникальным сочетанием свойств: по плотности и модулю Юнга магниевые сплавы наиболее близки к костной ткани человека и, что наиболее важно, они могут постепенно растворяться в организме, не причиняя ему вреда. В качестве основных перспективных сплавов для применения в медицине рассматриваются как уже известные системы $\mathrm{Mg}-\mathrm{Zn}-\mathrm{Zr}$ [3], так и новые $\mathrm{Mg}-\mathrm{Zn}-\mathrm{Ca}$ и Mg-Y-Zr [4]. Для внедрения и тех и других систем сплавов в медицинскую практику нужно решить две проблемы. Первая проблема связана с их недостаточно высокими прочностными и усталостными свойства, что особенно касается низколегированных систем типа $\mathrm{Mg}-\mathrm{Zn}-\mathrm{Ca}$. Эта проблема сейчас успешно решается за счет использования комплексной обработки, включающей такие передовые методы интенсивной пластической деформации, как изотермическая прокатка, ротационная ковка, всесторонняя изотермическая ковка, равноканальное угловое прессование и др. [5]. Однако вторая проблема, связанная с невысокими коррозионно-усталостными свойствами магниевых сплавов, до сих пор остается нерешенной. Основная сложность здесь заключается в том, что магний образует гальваническую пару практически с любыми элементами, в том числе, растворенными в самом сплаве. Как следствие, улучшение физико-механических свойств магния путем легирования в большинстве случаев приводит к ухудшению его коррозионных свойств, тем самым, в условиях коррозионной среды сводя на нет весь достигнутый эффект повышения усталостных свойств за счет твердорастворного упрочнения [6]. Когда же, помимо химической коррозии, реализуется электрохимическая, то долговечность магниевых сплавов на уровне предела выносливости $2 \times 10^{7}$ циклов вообще стремиться к нулю [7]. Кроме того, применение временных металлических конструкции в реконструктивной медицине предполагает, что с целью обеспечения оптимального режима остеосинтеза скорость процесса их саморассасывания в первые два-три месяца должна быть минимальной. Наиболее эффективным способом решения проблемы слишком низкой коррозионной стойкости (высокой скорости резорбции) магниевых сплавов является использование специальных защитных покрытий, которые ограничивали бы растворение магниевого сплава в первое время.

Разрабатываемые покрытия для магниевых сплавов условно можно разделить на металлические и неметаллические. Преимущество первых заключается в возможности получения тонких покрытий толщиной от доли микрона, а недостаток - в большой вероятности появления гальванической пары. Вторые, наоборот, исключают гальваническую пару, но сама технология нанесения сопряжена со значительными трудностями, требующими поиска их решений. Среди защитных металлических покрытий, наносимых на магниевые сплавы в вакууме (рассматривается наиболее чистая среда, что принципиально важно для медицинских изделий), наиболее разработанной являются технологии вакуумного алюминирования [8]. Однако алюминий токсичен для человеческого организма, в том числе, провоцирует развитие болезни Альцгеймера, накапливается в мозгу, вызывает ломкость костей и др. [9], и в связи с этим не может входить в состав биорезорбируемых материалов, используемых в медицине. Наиболее перспективными для практического применения видятся титановые и циркониевые покрытия, так как соединения на базе этих элементов уже активно используются в качестве постоянных имплантатов $[10,11]$. В представленной работе проводится комплексное исследование вакуумных циркониевых покрытий, наносимых на магниевый сплав MA14 (ZK60), который в настоящее время рассматривается как один из возможных кандидатов на использование в медицинской практике.

\section{2. Материалы и методы исследования}

Для исследования использовались прутки сплава MA14 с химическим составом, указанным в Табл. 1.

Покрытия наносились вакуумным ионно-плазменным методом на установке Булат-6К. Предварительно образцы обезжиривались нефрасом под воздействием ультразвука, а непосредственно перед постановкой в вакуумную камеру проводилась очистка дистиллиро- 
Табл. 1. Химический состав прутка сплава MA14 (аналог ZK60).

Table 1. Chemical compositions of the MA14 alloy (similar ZK60).

\begin{tabular}{|c|c|c|c|c|c|c|c|c|}
\hline Элемент / Element & $\mathrm{Zn}$ & $\mathrm{Zr}$ & $\mathrm{Al}$ & $\mathrm{Fe}$ & $\mathrm{Cu}$ & $\mathrm{Ni}$ & $\mathrm{Mn}$ & $\mathrm{Si}$ \\
\hline Среднее / Average & 5.693 & 0.860 & 0.001 & 0.004 & 0.002 & 0.004 & 0.005 & 0.008 \\
\hline $\begin{array}{c}\text { MA14 по ГОСТ 14957-76 } \\
\text { MA14 in GOST 14957-76 }\end{array}$ & $5.0-6.0$ & $0.3-0.9$ & $<0.05$ & $<0.03$ & $<0.05$ & $<0.005$ & $<0.1$ & $<0.05$ \\
\hline
\end{tabular}

ванной водой при помощи пароструйного аппарата под давлением 6 атм при температуре $120^{\circ} \mathrm{C}$. Для улучшения адгезии покрытия перед напылением был проведен объемный тлеющий разряд длительностью 10 минут в атмосфере аргона при давлении $8 \times 10^{-3}$ мм.рт.ст. Процесс напыления проводился с одного катода бокового расположения при давлении аргона $5 \times 10^{-5}$ мм. рт.ст. В качестве напыляемого материала использовался высокочистый цирконий номинального содержания 99.99\%. Для обеспечения равномерности покрытия по всей площади образцы были размещены на рабочем столе с подачей вращения 20 об/мин. Для уменьшения травления поверхности потенциал на рабочий стол не подавался. Время напыления варьировалось для получения покрытий заданной толщины.

Качество покрытий оценивалось с помощью скрэтчтестера Nanovea P-Macro. Для нанесения царапин использовался индентор конической формы со сферической вершиной радиусом 100 мкм. Испытание проводилось по следующей методике: царапина длиной 10 мм наносилась путем линейного увеличения вертикальной нагрузки на индентор от 0.1 до $2 \mathrm{H}$ и перемещения предметного столика с образцом со скоростью 10 мм/мин.

Испытание на коррозионную стойкость образцов с нанесенными покрытиями проводилось в среде раствора Рингера (аналог 0.9\% $\mathrm{NaCl}$ ) на специально собранной установке (Рис. 1) по выходу водорода с единицы поверхности. В камере с помощью перистальтического насоса LOIP LS-301 и водяной бани LOIP LT-411b поддерживалась постоянная температура $37 \pm 1^{\circ} \mathrm{C}$. $\mathrm{Ph}$ раствора контролировался с помощью лабораторного ионометра И-150МИ и поддерживался в узком диапазоне путем автоматического впрыскивания разбавленной ортофосфорной кислоты.

Металлографические исследования и определение толщины полученных покрытий проводилось на лазерном конфокальном микроскопе Olympus LEXT OLS4000 и сканирующем электронном микроскопе Zeiss Sigma. Удаление продуктов коррозии осуществлялось путем погружения в раствор $20 \% \mathrm{CrO}_{3}+1 \% \mathrm{AgNO}_{3}$ (ГОСТ Р 9.907-2007) с последующим промыванием в изопропиловом спирте и сушкой сжатым воздухом.

\section{3. Результаты и их обсуждение}

Внешний вид циркониевых покрытий, полученный с использованием сканирующего электронного микроскопа, показан на Рис. 2.

Толщина полученных покрытий составила $0.4 \pm 0.2$ мкм (Рис. 2 a) и $0.8 \pm 0.2$ мкм (Рис. 2 b), соответственно. Можно заметить практически полное отсутствие диффузионного слоя между самим покрытием и подложкой, что связанно с невысокой температурой разогрева основы с целью

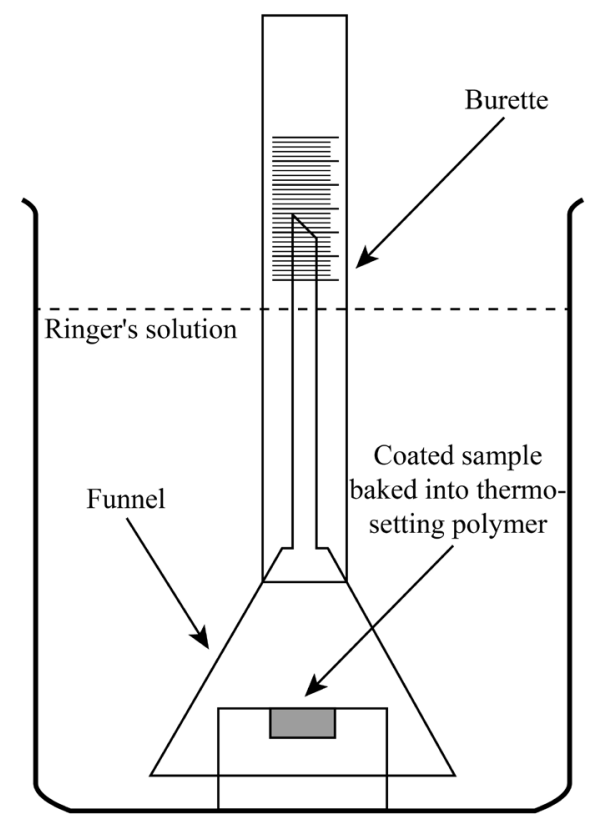

Рис. 1. Принципиальная схема установки по оценке коррозионной стойкости магниевых сплавов по выходу водорода.

Fig. 1. Schematic diagram of the equipment for testing of the corrosion resistance of magnesium alloys in terms of hydrogen evolution.

недопущения ухудшения физико-механических свойств (нагрев сплава MA14 выше $250^{\circ} \mathrm{C}$ приводит к значимому падению предела текучести и прочности). Необходимо отметить, что изготовление шлифов для таких тонких покрытий сопряжено со значительными трудностями, из-за высокой вероятности повреждения покрытия при полировке или нарушения его адгезии к подложке при запрессовывании в реактопласт. Проведенный локальный химический анализ подложки (Рис. 3 a) и покрытия (Рис. $3 \mathrm{~b}$ ) подтвердил, что покрытие состоит преимущественно из циркония.

Для проверки связи покрытия с подложкой, а также оценки типа разрушения, были проведены испытания по царапанию для покрытия толщиной $0.8 \pm 0.2$ мкм (Рис. 4).

По полученным изображениям можно заметить, что в процессе теста индентор начинает продирать покрытие при нагрузке вдавливания порядка 0.5 Н (Рис. 4), a наличие характерных сколов после прохождения индентора свидетельствует о том, что покрытие является более хрупким по сравнению с вязкой основой, на которую оно было нанесено.

В настоящее время разработано несколько методов, по оценке коррозионной повреждаемости магниевых сплавов: по убыли массы, потенциостатический, по количеству выделившегося водорода [12-14]. Для 


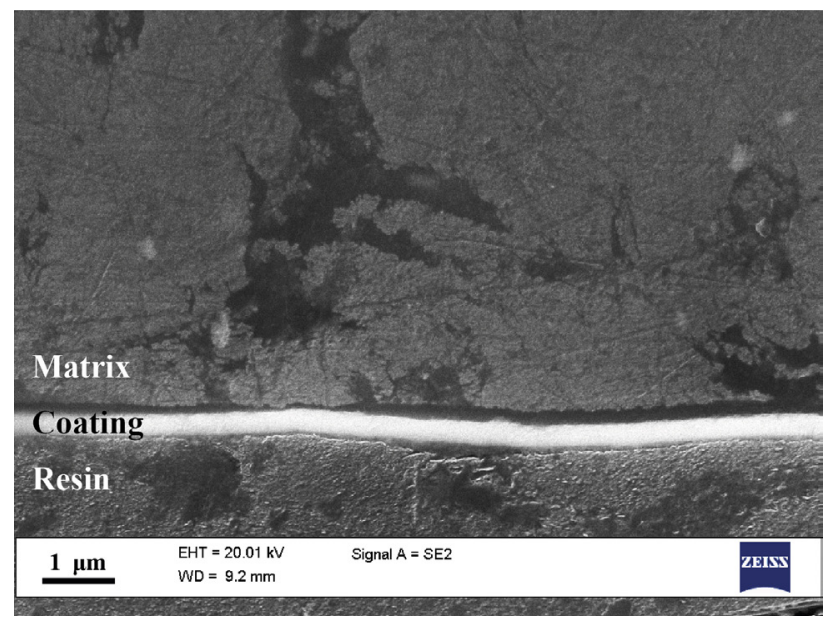

a

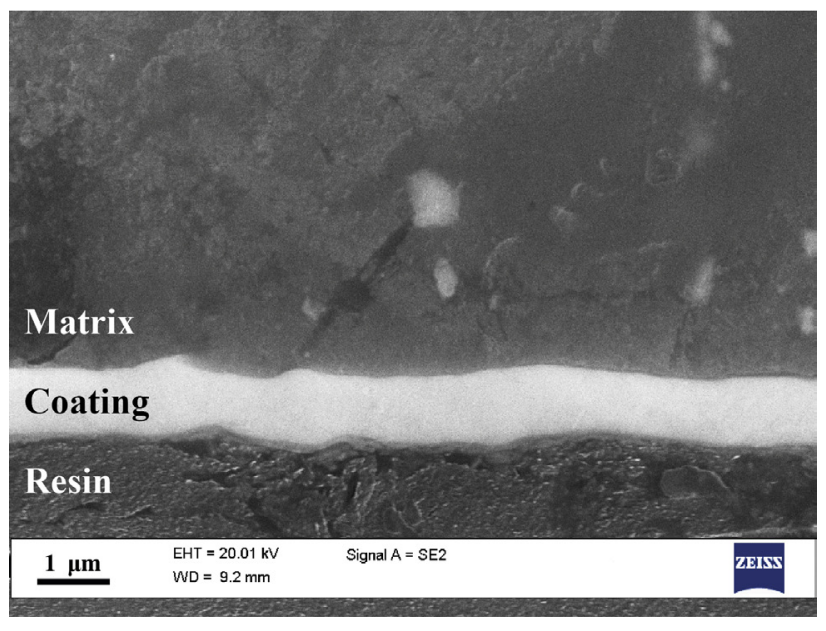

$\mathrm{b}$

Pис. 2. СЭМ изображения $\mathrm{Zr}$ покрытий толщиной $0.4 \pm 0.2$ мкм (а) и $0.8 \pm 0.2$ мкм (b).

Fig. 2. SEM images of $\mathrm{Zr}$ coatings with a thickness $0.4 \pm 0.2 \mu \mathrm{m}$ (a) and $0.8 \pm 0.2 \mu \mathrm{m}$ (b).

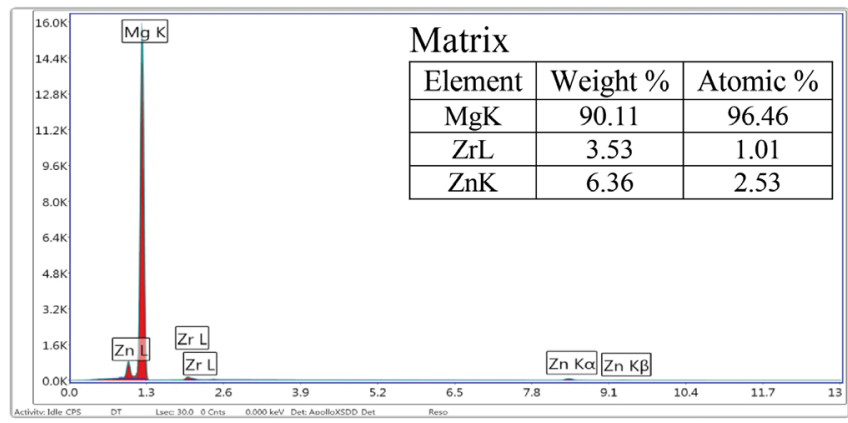

a

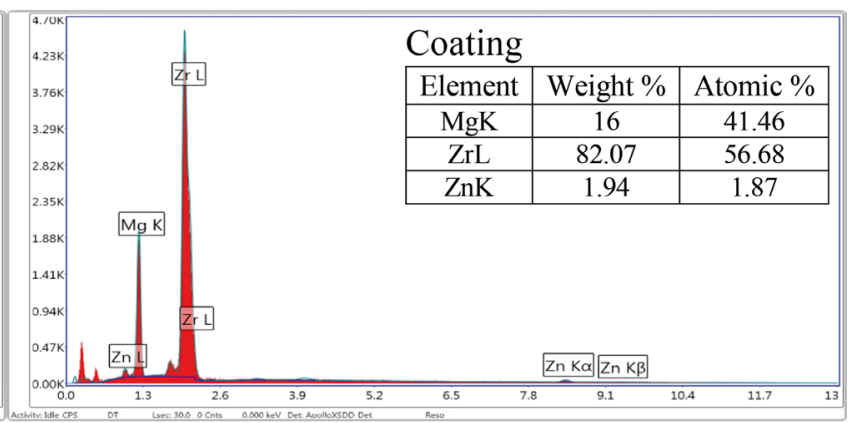

$\mathrm{b}$

Рис. 3. Результаты спектрального анализа X-ray (EDX) магниевой матрицы (a); и циркониевого покрытия (b). Fig. 3. Energy Dispersive X-ray (EDX) spectra obtained from the a-Mg matrix (a); and the zirconium coating (b).

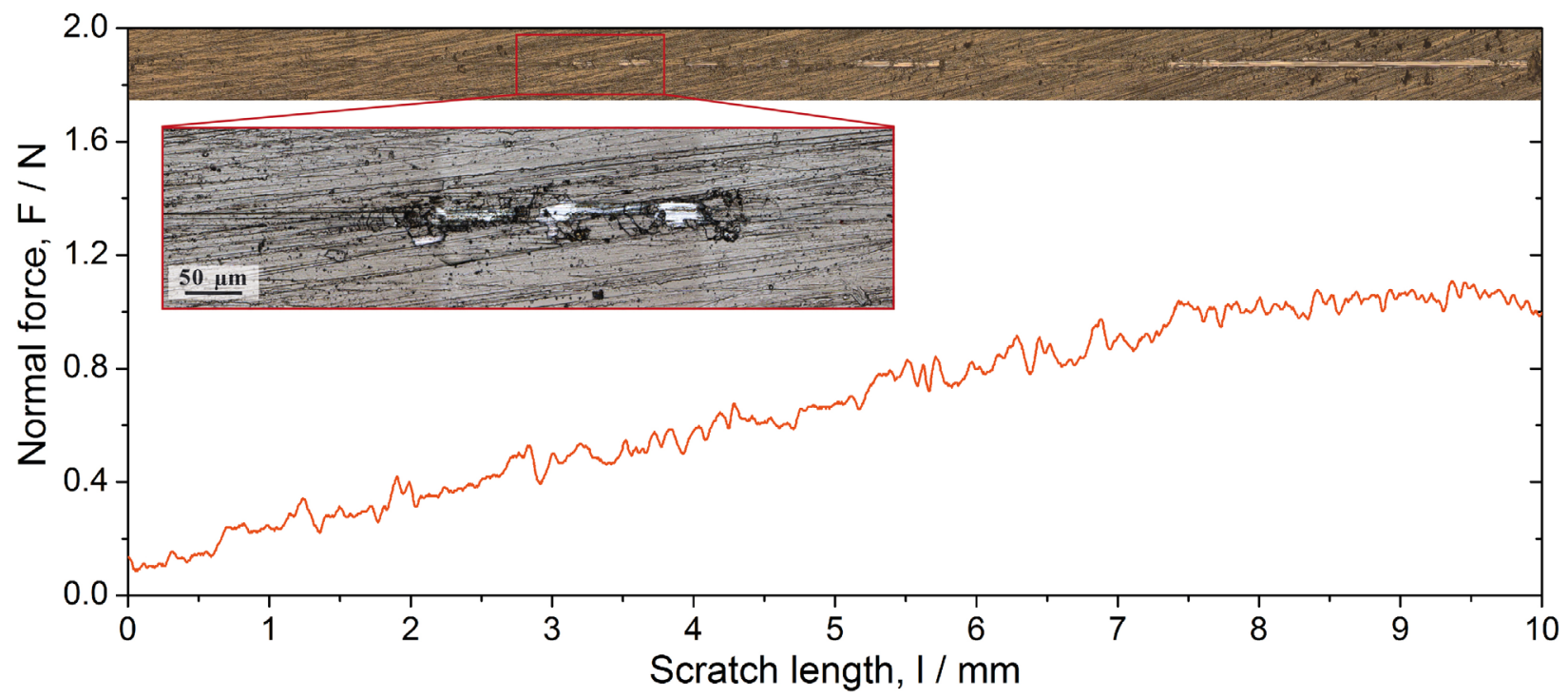

Pис. 4. (Color online) Типичная царапина, нагрузка вдавливания, а также увеличенное изображение момента отслаивания покрытия после испытания образца сплава MA14, покрытого Zr толщиной $0.8 \pm 0.2$ мкм.

Fig. 4. (Color online) Typical scratch, indentation load, as well as an enlarged image of the moment of coating peeling, obtained after testing a sample of magnesium alloy MA14 coated with $\mathrm{Zr}$ with a thickness of $0.8 \pm 0.2 \mu \mathrm{m}$. 
оценки защитных свойств покрытий в данной работе был выбран последний метод, т.к. только он позволяет проследить кинетику изменения скорости коррозионной повреждаемости в реальном времени. Типичные кривые количества выделившегося водорода, приведенного к единице площади поверхности, а также возможные химические реакции, протекающие на поверхности чистого магния в коррозионной среде, показаны на Рис. 5.

Скорость коррозии вычислялась по формуле:

$$
V_{\text {cor }}=\frac{8.76 \cdot 10^{4} \cdot W}{A \cdot T \cdot D}
$$

где $W-$ потеря веса в г, $A-$ площадь поверхности образца в см ${ }^{2}, T-$ время выдержки в коррозионной среде в часах, $D-$ плотность металла в г/ см$^{3}$.

При этом потеря веса пересчитывалась в количество вещества исходя из того, что 1 моль выделяющегося в процессе реакции (Рис. 5) водорода соответствует 1 молю растворившегося магния. Полученные таким образом скорости коррозии в зависимости от времени выдержки в растворе Рингера занесены в Табл. 2.

Нанесение циркониевого покрытия толщиной 0.4 мкм способствует статистически значимому уменьшению скорости коррозии примерно через 80 часов испытания, а толщиной 0.8 мкм - уже после 60 часов (Табл. 2). Циркониевые покрытия толщинами 0.4 и 0.8 мкм на базе испытания, равной 110 часам (4.5 суток), уменьшают скорость коррозии в 1.3 и 1.6 раза соответственно по сравнению с образцами без покрытия. Полученные результаты хорошо согласуются с рельефом поверхности образцов, снятым с помощью лазерного конфокального микроскопа после удаления продуктов коррозии (Рис. 6): наблюдается уменьшение как общей прокорродировавшей площади (что особенно заметно на образце, покрытом цирконием толщиной 0.8 мкм), так и глубины коррозионных повреждений.

\section{4. Заключение}

Циркониевое покрытие толщиной 0.8 мкм обеспечивает снижение скорости коррозии сплава MA14 (ZK60) в физиологическом растворе Рингера на базе экспозиции 110 часов в 1.6 раза. Таким образом, применение подобных покрытий позволяет, во-первых, повысить долговечность работы изделий из магниевых сплавов, во-вторых, реализовать переменную скорость резорбции: пониженную в первоначальный период и большую (равную скорости растворения основы) в последующий (после прекращения защитных свойств покрытия) и, в-третьих, регулировать скорость резорбции и долговечность магниевых сплавов путем варьирования толщины циркониевых покрытий.

Табл. 2. Скорость коррозии по выходу водорода.

Table 2. Corrosion rate assessed by the hydrogen evolution method.

\begin{tabular}{|c|c|c|c|c|c|c|}
\hline \multirow{2}{*}{$\begin{array}{l}\text { Время, час } \\
\text { Time, h }\end{array}$} & \multicolumn{6}{|c|}{$\begin{array}{l}\text { Скорость коррозии (глубинный показатель), мм/год* } \\
\text { Corrosion rate (depth index), mm/year* }\end{array}$} \\
\hline & 20 & 40 & 60 & 80 & 100 & 110 \\
\hline $\begin{array}{l}\text { Без покрытия } \\
\text { Without coating }\end{array}$ & 4.5 & 4.2 & 5.6 & 8.3 & 12.7 & 13.8 \\
\hline $\begin{array}{c}\text { Покрытие Zr } 0.4 \pm 0.2 \text { мкм } \\
\text { Coating Zr } 0.4 \pm 0.2 \mu \mathrm{m}\end{array}$ & 4.2 & 4.9 & 5.5 & 6.4 & 9.4 & 10.5 \\
\hline $\begin{array}{c}\text { Покрытие Zr } 0.8 \pm 0.2 \text { мкм } \\
\text { Coating Zr } 0.8 \pm 0.2 \mu \mathrm{m}\end{array}$ & 3.7 & 3.4 & 3.9 & 5.3 & 7.8 & 8.5 \\
\hline
\end{tabular}

${ }^{*}$ средняя погрешность определения скорости коррозии, вычисленная по трем образцам, составляет \pm 1.2 мм/год

${ }^{\star}$ the average error in determining the corrosion rate, calculated from three samples, is $\pm 1.2 \mathrm{~mm} /$ year

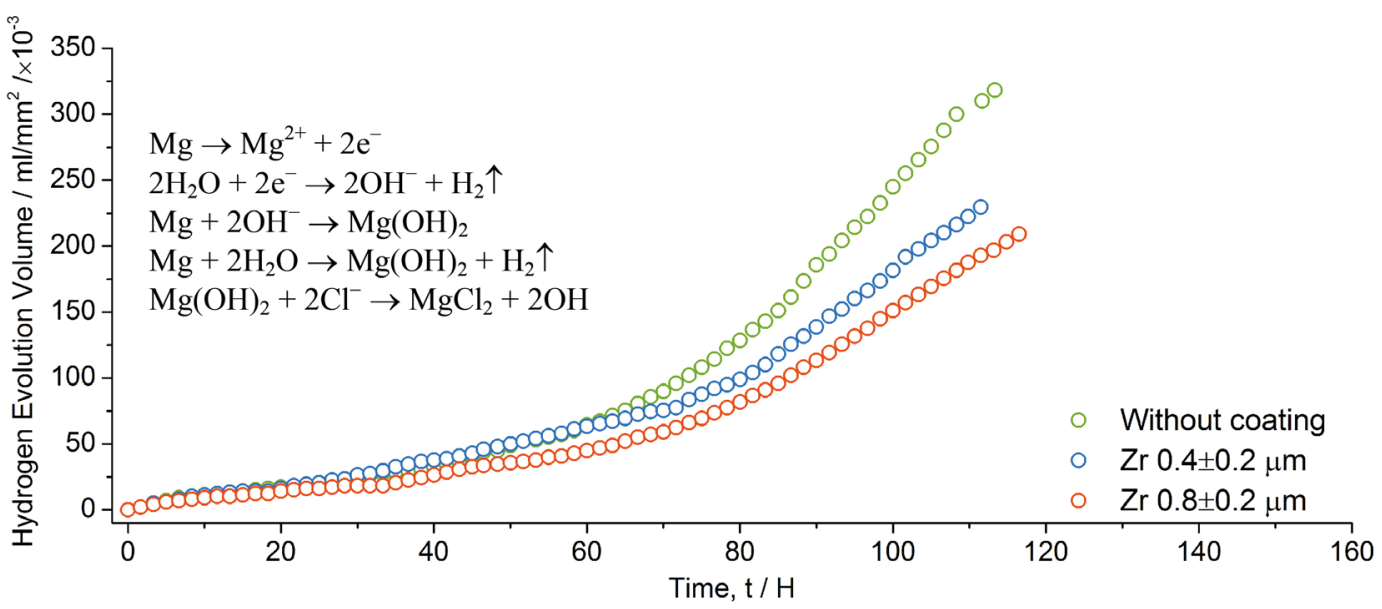

Pис. 5. (Color online) Кривые выхода водорода для образцов магниевого сплава MA14 в исходном состоянии и покрытых Zr толщиной $0.4 \pm 0.2$ мкм и $0.8 \pm 0.2$ мкм.

Fig. 5. (Color online) Hydrogen evolution curves for the magnesium alloys MA14 in the initial state and coated with Zr with a thickness of $0.4 \pm 0.2 \mu \mathrm{m}$ and $0.8 \pm 0.2 \mu \mathrm{m}$. 


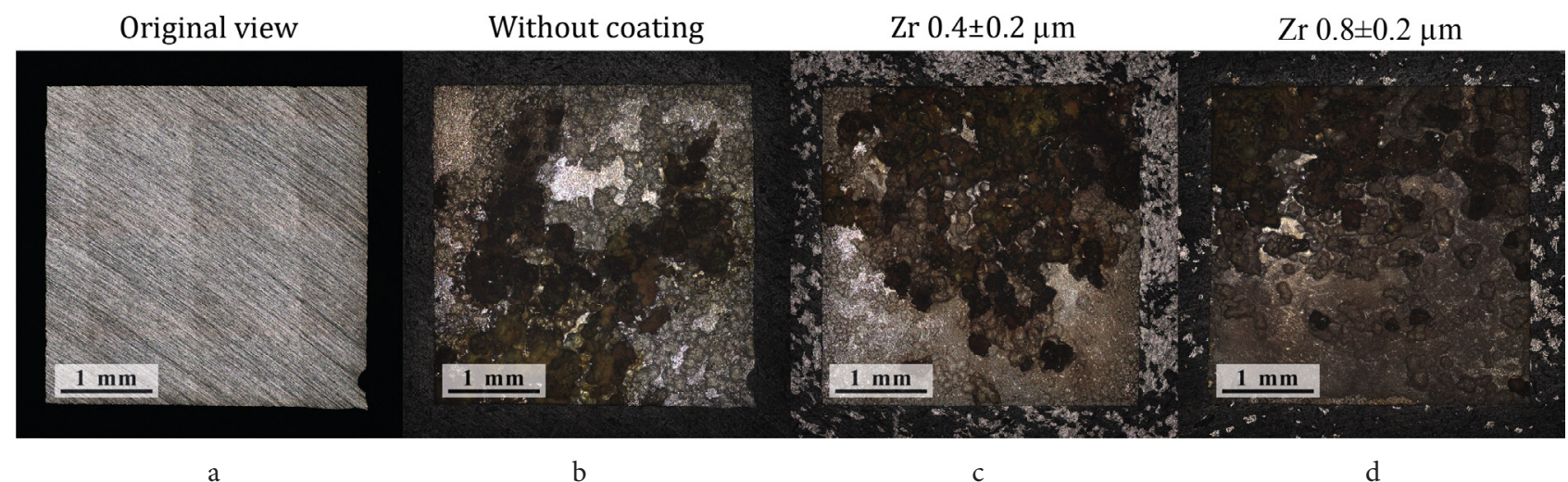

Pис. 6. (Color online) Морфология образцов магниевого сплава MA14 в исходном состоянии и покрытых Zr, после выдержки в среде Рингера в течение 110 часов и удалении продуктов коррозии.

Fig. 6. (Color online) Optical macro-morphologies of the MA14 specimens in the initial state and coated with Zr, after immersing in Ringer $\mathrm{SBF}$ for $110 \mathrm{~h}$ and corrosion product removal.

Благодарности / Acknowledgments. Исстедование выполнено за счет гранта Российского научного фонда (проект № 19-79-00258). / The present work was supported by the Russian Science Foundation (project № 19-79-00258).

\section{Литература/References}

1. P. Jiang, C. Blawert, M.L. Zheludkevich. Corros. Mater. Degrad. 1 (2), 7 (2020). Crossref

2. U. Riaz, I. Shabib, W. Haider. J. Biomed. Mater. Res. B. 107 (6), 1970 (2019). Crossref

3. Y. Zhang, N. Wan, J. Li, M. Mesbah, K. Wong, A. Fallahpour, B. Nasiri-Tabrizi, J. Yang. Lat. Am. j. solids struct. 17 (5), e293 (2020). Crossref

4. D. Merson, A. Brilevsky, P. Myagkikh, A. Tarkova, A. Prokhorikhin, E. Kretov, T. Frolova, A. Vinogradov. Materials. 13 (3), 544 (2020). Crossref

5. E. Vasilev, V. Kopylov, M. Linderov, A. Brilevsky, D. Merson, A. Vinogradov. Letters on Materials. 9 (2), 157 (2019). Crossref

6. A. Vinogradov, E. Vasilev, V. Kopylov, M. Linderov, A. Brilevesky, D. Merson. Metals. 9 (2), 186 (2019). Crossref

7. M. Linderov, E. Vasilev, D. Merson, M. Markushev, A. Vinogradov. Metals. 8 (1), 20 (2018). Crossref
8. I. Roikh, L. Koltunova, S. Fedosov. Vacuum protective coating. Moscow (1976) 369 p. (in Russian) [И. Ройх, Л. Колтунова, С. Федосов. Нанесение защитных покрытий в вакууме. Москва (1976) 369 с.]

9. L. Morozova. Chemical elements in the human body: reference materials. Arkhangelsk, Federal University named after M.V. Lomonosov (2001) 47 p. (in Russian) [Л.В. Морозова. Химические элементы в организме человека: справочные материалы. Архангельск, Поморский государственный университет им. М. В. Ломоносова (2001) 47 с.]

10. G. Renganathan, N. Tanneru, S. Madurai. Fundamental Biomaterials: Metals. 2018, 211 (2018). Crossref

11. M. Ferraris, E. Verne, P. Appendino, C. Moisescu, A. Krajewski, A. Ravaglioli, A. Piancastelli. Biomaterials. 21 (8), 765 (2000). Crossref

12. M. Esmaily, J. Svensson, S. Fajardo, N. Birbilis, G. Frankel, S. Virtanen, R. Arrabal, S. Thomas, L. Johansson. Progress in Materials Science. 89 (2169), 92 (2017). $\underline{\text { Crossref }}$

13. S. Lebouil, A. Duboin, F. Monti, P. Tabeling, K. Ogle, P. Volovitch. Electrochimica Acta. 124, 276 (2013). Crossref

14. G. Frankel. Journal of Testing and Evaluation. 42 (3), 517 (2014). $\underline{\text { Crossref }}$ 